\title{
SÍNTESE DE NANOPARTÍCULAS DE OURO E SENSORIAMENTO COLORIMÉTRICO DO SULFATIAZOL: UMA PRÁTICA EXPERIMENTAL PARA AULAS INTERDISCIPLINARES
}

\author{
Elias Barros Santos ${ }^{\mathrm{a}, \#}$, Letícia Cuesta Matos $^{\mathrm{a}}$ e Italo Odone Mazali ${ }^{\mathrm{b}, *,(\mathbb{0}}$ \\ 'Laboratório de Química Ambiental e Nanomateriais, Universidade Federal de São Paulo, 12231-280, São José do Campos - SP, Brasil \\ 'Laboratório de Materiais Funcionais, Instituto de Química, Universidade Estadual de Campinas, 13083-970 Campinas - SP, Brasil
}

Recebido em 14/09/2021; aceito em 06/12/2021; publicado na web em 27/01/2022

\begin{abstract}
SYNTHESIS OF GOLD NANOPARTICLES AND COLORIMETRIC SENSING OF THE SULFATHIAZOLE: AN EXPERIMENTAL PRACTICE FOR INTERDISCIPLINARY CLASSES. Students undertaking courses in the field of sciences and engineering need to learn practical skills and conceptual knowledge, and the experimental classes are the best moment for this. In this paper, we propose a practical experiment to aid nanotechnology learning by synthesizing gold nanoparticles (AuNPs) and their application as a colorimetric sensor of sulfathiazole. AuNPs were prepared using sodium citrate as a reducing and capping agent. The existence of the synthesized AuNPs was detected using the Tyndall effect while their optical properties were investigated by molecular absorption spectrophotometry. The gold nanoparticles exhibit spherical-like shape morphology and an average size of $13.6 \mathrm{~nm}$, determined by transmission electron microscopy. The AuNPs were applied as a colorimetric sensor for the detection of sulfathiazole in mixed water/ethanol solution by simple visual inspection and using molecular absorption spectrophotometry. Due to the simplicity of the experimental steps, this activity is well-suited for a hands-on introduction of nanoparticles synthesis and its application as a molecular sensor. In addition, the experiment encompasses knowledge from different fields being suitable for interdisciplinary classes.
\end{abstract}

Keywords: nanotechnology; interdisciplinary; gold nanoparticles; colorimetric sensor; higher education.

\section{INTRODUÇÃO}

As práticas experimentais são extremamente importantes e indispensáveis para a formação de vários profissionais, como, por exemplo, químicos, biólogos, físicos, engenheiros, médicos, farmacêuticos, etc. As aulas experimentais permitem aos estudantes vivenciar casos reais ou simular atividades cotidianas da profissão que estão almejando, resultando em um aprendizado único e diferenciado das disciplinas teóricas. A experimentação permite o dinamismo da aula, estimula o trabalho em equipe, acontece em um ambiente diferente (geralmente laboratórios ou como atividades de campo), facilita o aprendizado de habilidades práticas, sendo muito valorizada pelos estudantes e gerando expectativas entre os mesmos. Entretanto, a prática experimental que meramente repete roteiros passa a ser cansativa e desestimulante, além de ainda manter os estudantes com dúvidas, principalmente por apenas terem executado uma ação automática com resultado esperado. ${ }^{1}$ Lima e Alves, relataram uma pesquisa realizada com estudantes do ensino médio sobre um ensino de química mais satisfatório. Os entrevistados responderam que para tal finalidade, o ensino de química precisa ser realizado com mais aulas experimentais, pois tornam esta ciência mais interessante e motivadora, e também necessita de mais contextualização, com aulas apresentando maior clareza e objetividade. ${ }^{2}$ Nesse sentido, e dada a relevância da prática experimental na formação dos futuros profissionais, as aulas experimentais em qualquer nível de formação devem ser mais desafiadoras e interdisciplinares, possibilitando aos estudantes vivenciar o problema e trabalharem na busca da melhor solução.

A interdisciplinaridade é um conceito que busca a intersecção entre conteúdos de duas ou mais disciplinas para permitir que o estudante elabore uma visão mais ampla a respeito das temáticas. ${ }^{3}$ Nessa direção, o conteúdo programático dos cursos passa a ser

*e-mail: mazali@unicamp.br

\#e-mail alternativo: santos.barros@unifesp.br integrado em disciplinas com caráter interdisciplinar ao invés das tradicionais divisões em disciplinas isoladas. A prática da interdisciplinaridade pode enriquecer, em vários aspectos, a qualidade das atividades experimentais, permitindo aos estudantes assimilarem uma visão mais ampla das questões abordadas em aula. Os problemas diários do mercado de trabalho e as questões globais estão integrados, como bem exemplificado pela Organização das Nações Unidas nos 17 objetivos de desenvolvimento sustentável. ${ }^{4}$ Consequentemente, os profissionais precisam passar por um processo de formação que os prepare para a vida real. Por exemplo, com o surgimento da nanotecnologia e sua popularização crescente para diversas áreas do conhecimento, nota-se a integração de conteúdos em uma única área. A nanotecnologia é um campo multidisciplinar, que integra várias disciplinas fundamentais, como por exemplo, física, química, matemática e biologia, alcançando as engenharias, medicina, indústria alimentícia, etc. ${ }^{5}$ Em outras palavras, em uma atividade didática com o tema central "Nanotecnologia", vários conteúdos de outras disciplinas podem ser integrados no sentido de se atingir uma compreensão mais ampla.

A nanotecnologia é uma área do conhecimento que engloba ciência, engenharia e tecnologias baseadas em materiais fabricados na nanoescala, entre 1 e $100 \mathrm{~nm} .{ }^{6}$ Os materiais quando reduzidos a essa escala de tamanho apresentam propriedades diferenciadas da sua contraparte micro e macro, devido à alta razão área de superfície/ volume e aos efeitos de confinamento quântico por tamanho. ${ }^{6,7}$ Com isso, é possível aplicá-los na geração de novas tecnologias ou aperfeiçoar tecnologias já existentes, resultando na denominada nanotecnologia. Para uma melhor compreensão e entendimento dos fenômenos da matéria em nanoescala é de fundamental importância estudar a ciência das nanopartículas. Por exemplo, o ouro em escala macro apresenta cor amarela e brilho metálico característico. Entretanto, esse mesmo elemento na forma de nanopartículas, com dimensão inferior a $100 \mathrm{~nm}$, pode exibir outras cores, dependentes do tamanho, da morfologia e do meio ao qual as partículas estão imersas. ${ }^{89}$ Essa mudança de propriedade tem origem na ressonância 
plasmônica de superfície (SPR, do inglês surface plasmon resonance), resultante de variações na interação das nanopartículas com a radiação eletromagnética incidente. Essas variações ocorrem devido a mudanças na dinâmica coletiva dos elétrons, que oscilam em diferentes frequências dependentes do tamanho, morfologia e constante dielétrica do meio. ${ }^{8,9}$ Consequentemente, as nanopartículas de ouro podem exibir diferentes cores (vermelho, azul, violeta), resultantes de variações do espalhamento da radiação incidente. ${ }^{10}$

O sulfatiazol foi escolhido como objeto de estudo devido a sua característica química, apresentando grupos funcionais com átomos de nitrogênio e enxofre, os quais apresentam afinidade química com o ouro. Por ser um composto de origem farmacêutica usado como antibiótico para infecções bacterianas humana e veterinária, o sulfatiazol também já foi relatado como contaminante emergente. ${ }^{11,12}$ A presença desses compostos em água pode ser nociva não somente a saúde humana, mas também à biodiversidade do ambiente, sendo o seu monitoramento e destino de interesse ambiental. ${ }^{13}$ Este assunto pode ser abordado pelo docente com os discentes, contextualizando a prática experimental com um tema cotidiano e também mostrando como a nanotecnologia pode ser empregada na solução do problema. Além disso, existem poucos trabalhos na literatura envolvendo a interação do sulfatiazol com nanoestruturas de ouro, sendo os principais relacionados a detecção por SERS (surface-enhanced Raman spectroscopy) e sensoriamento eletroquímico. ${ }^{14,15}$ Esse fato também indica que o presente trabalho apresenta uma proposta inédita.

Diante do exposto, o objetivo do presente trabalho é apresentar um experimento de síntese de nanopartículas de ouro e sua aplicação no sensoriamento colorimétrico do sulfatiazol. O experimento é apresentado como uma proposta de prática experimental interdisciplinar, que engloba conhecimentos de síntese química, físico-química de superfície e coloidal, fármacos e análise ambiental, contextualizada com a nanotecnologia. Além disso, o presente trabalho também engloba aspectos da nanoquímica, que é um termo usado no contexto da nanotecnologia para enfatizar os aspectos relacionados à síntese de nanoestruturas e estudo das suas propriedades químicas. ${ }^{16}$ Geoffry Ozin, um pioneiro no campo da nanoquímica, descreveu em seu livro intitulado "Nanochemistry: A chemical approach to nanomaterials", a importância do estudo dos aspectos químicos dos nanomateriais para uma melhor compreensão das propriedades físico-químicas e aplicação. ${ }^{16}$

\section{PARTE EXPERIMENTAL}

Em disciplinas experimentais, é muito comum o docente realizar uma discussão inicial com os discentes antes de começar a prática experimental. Nesse momento, é importante que os discentes entendam, em linhas gerais, o objetivo do experimento e as etapas que precisam ser executadas para atingi-lo. $\mathrm{O}$ uso de fluxogramas é uma estratégia didática eficiente adotada pelos docentes e muitas vezes solicitada pelos próprios discentes. Nesse sentido, para a prática experimental apresentada neste artigo, sugere-se usar o fluxograma mostrado na Figura 1, no qual são descritas as três etapas principais do experimento de forma resumida e que também poderá ser adaptado pelo docente. Com esse fluxograma, os discentes identificarão rapidamente quais são os reagentes necessários, as soluções químicas que precisarão preparar, as análises que farão para caracterizar as nanopartículas de ouro (AuNPs) e os testes de detecção do sulfatiazol.

\section{Reagentes usados no experimento}

Os reagentes ácido cloroáurico $(99,99 \%)$, citrato de sódio tribásico ( $\geq 99,0 \%)$, sulfatiazol (99,0\%), álcool etílico (95,0\%), ácido

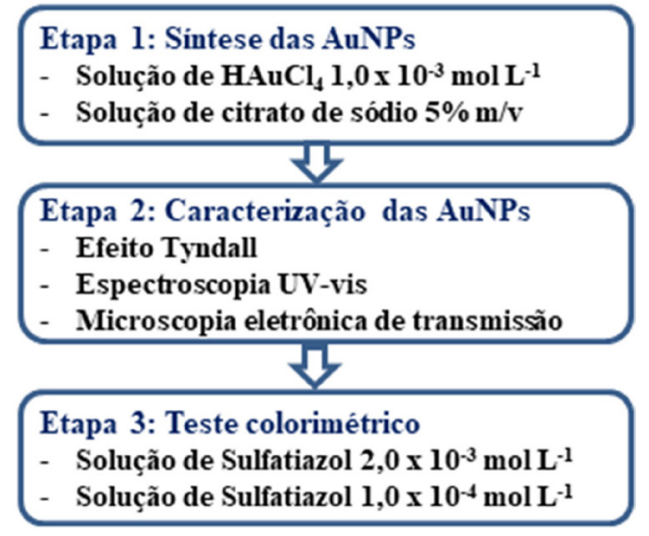

Figura 1. Fluxograma didático resumindo as três etapas do procedimento experimental

sulfúrico $(95,0$ - 98,0\%) e peróxido de hidrogênio $(30,0 \%)$ foram adquiridos da Sigma-Aldrich e usados como recebidos.

\section{Primeira etapa: síntese das nanopartículas de ouro}

O primeiro procedimento foi o preparo das soluções usadas no experimento. Foi preparado $50 \mathrm{~mL}$ de uma solução aquosa de ácido cloroáurico $\left(\mathrm{HAuCl}_{4}\right)$ na concentração de $1,0 \times 10^{-3} \mathrm{~mol} \mathrm{~L}^{-1}$, reagente usado como precursor de ouro. Foi preparada uma solução aquosa de citrato de sódio na concentração de $5 \% \mathrm{~m} / \mathrm{v}$, reagente usado como agente redutor e estabilizante. Foi também preparada uma solução piranha (mistura de ácido sulfúrico e peróxido de hidrogênio 4:1 v/v) para a limpeza da vidraria usada na síntese.

O coloide de nanopartículas de ouro (AuNPs) foi sintetizado de acordo com um método já reportado na literatura, com algumas adaptações. ${ }^{17} \mathrm{~A}$ solução de $\mathrm{HAuCl}_{4}$ foi transferida para um erlenmeyer de $100 \mathrm{~mL}$, sendo mantida sob agitação constante e aquecimento até atingir ebulição. Nessa condição, foi adicionado $1 \mathrm{~mL}$ da solução de citrato de sódio $5 \% \mathrm{~m} / \mathrm{v}$. Após $\sim 5 \mathrm{~min}$, a mistura mudou de cor, passando do amarelo característico do $\mathrm{HAuCl}_{4}$ para o vermelho vinho. $\mathrm{O}$ aquecimento foi interrompido após $15 \mathrm{~min}$ da mudança de cor, mas a agitação foi mantida até o resfriamento do coloide.

Em uma aula experimental, o volume de $50 \mathrm{~mL}$ do coloide de AuNPs é suficiente para atender dez grupos de estudantes, uma vez que no teste de sensoriamento do sulfatiazol foram usados $5,0 \mathrm{~mL}$ no total, como descrito na terceira etapa abaixo.

\section{Segunda etapa: caracterização das AuNPs}

O coloide de AuNPs foi analisado após a síntese usando um laser com radiação verde para evidenciar o efeito Tyndall. ${ }^{18}$

Os espectros de absorção foram coletados usando um espectrofotômetro JASCO, modelo V-730-iRM, na faixa de 200 a $800 \mathrm{~nm}$. Uma parte do coloide foi transferida para uma cubeta de quartzo de caminho óptico igual a $1 \mathrm{~cm}$, sendo em seguida registrado o seu espectro.

As imagens de microscopia eletrônica de transmissão (TEM) foram obtidas usando o microscópio da JEOL, modelo JEM-2100 (200 kV de potência e $0.25 \mathrm{~nm}$ de resolução pontual). A amostra para TEM foi preparada por deposição de gotas do coloide de AuNPs sobre grade de cobre, recoberta com filme fino de carbono. Após a evaporação da água, a amostra foi analisada. A contagem de tamanho de partículas foi realizada a partir da análise das imagens de TEM, usando o software ImageJ, que é um programa de livre acesso disponível para download na internet. Os valores foram transferidos para uma planilha, sendo gerado o histograma de distribuição de tamanho. 
Vale ressaltar aqui, que a análise de microscopia eletrônica não é obrigatória como parte da prática experimental, visto que este procedimento de síntese é reprodutível. A escolha fica a critério do docente, levando em consideração a disponibilidade e acessibilidade ao microscópio em sua instituição. Caso o docente não realize essa caracterização, recomenda-se consultar referências bibliográficas que relatem esse tipo de análise, como as referências 19 e 20, e o assunto seja discutido com os discentes. ${ }^{19,20}$ Discutir, por exemplo, como é realizada a caracterização por TEM e como interpretar as micrografias coletadas. Os leitores também podem acessar imagens de TEM das AuNPs sintetizadas neste trabalho em informação suplementar.

\section{Terceira etapa: sensoriamento colorimétrico do sulfatiazol}

Para o sensoriamento colorimétrico, foram preparadas duas soluções de sulfatiazol nas concentrações $2,0 \times 10^{-3}$ e $1,0 \times 10^{-4} \mathrm{~mol} \mathrm{~L}^{-1}$, usando etanol como solvente devido à baixa solubilidade do composto em água. Em seguida, foi coletado $1 \mathrm{~mL}$ da solução mais concentrada e transferido para uma cubeta de quartzo contendo $2,5 \mathrm{~mL}$ do coloide de AuNPs, resultando em uma concentração final de 5,7 x $10^{-4} \mathrm{~mol} \mathrm{~L}^{-1}$. A cubeta contendo a mistura foi fotografada e posicionada no porta amostra do espectrofotômetro, sendo coletado espectros de absorção em diferentes tempos (1, 25, 50, 75 e 100 min). Após esse tempo, a cubeta foi retirada do equipamento e fotografada para comparação. O mesmo procedimento foi realizado para a segunda solução de sulfatiazol, que resultou em uma concentração final de $2,9 \times 10^{-5} \mathrm{~mol} \mathrm{~L}^{-1}$ após a correção do efeito de diluição.

\section{Manejo dos resíduos gerados}

Após finalizar a prática experimental, os $7 \mathrm{~mL}$ da mistura do coloide de AuNPs com sulfatiazol foi transferido para um recipiente de vidro, identificado e armazenado no abrigo de resíduos do laboratório. Após várias execuções desse procedimento e acúmulo de maior volume, o resíduo poderá ser tratado com água régia (mistura de ácido clorídrico e ácido nítrico), para recuperação do ácido cloroáurico que poderá ser reaproveitado em outra síntese de nanopartículas de ouro. ${ }^{21}$

Para o procedimento de recuperação do ouro, sugere-se centrifugar as AuNPs e remover o sobrenadante, que contém citrato de sódio como primeiro estabilizante. Suspender as AuNPs em água destilada, que estarão com sulfatizol como estabilizante, e adicionar água região na proporção $3 \mathrm{HNO}_{3}: 4 \mathrm{HCl}$. Nessa proporção, o ouro é oxidado, gerando ácido cloroáurico, água e gás dióxido de nitrogênio, que irá se desprender da solução. Para separar o sulfatiazol do meio, como este composto é pouco solúvel em água, pode ser realizada uma extração líquido-líquido, usando solventes orgânicos, como etanol ou acetona.

\section{RESULTADOS E DISCUSSÃO}

Na Figura 2 é apresentado o espectro de absorção na região do visível do coloide de ouro após a síntese. Observa-se uma banda de absorção com máximo em $520 \mathrm{~nm}$, referente a ressonância plasmônica de superfície característica de nanopartículas de ouro. ${ }^{9}$ Outra forte evidência da formação de ouro nanométrico é a cor vermelha observada no coloide, comumente relatada na literatura científica para nanopartículas de ouro. ${ }^{8,9}$

Na Figura 3(a) e (b), são apresentadas fotografias mostrando a incidência do laser em água destilada (na qual nenhum efeito foi observado) e no coloíde de AuNPs, no qual o efeito Tyndall, típico de um sistema coloidal é observado, respectivamente. Esse efeito tem

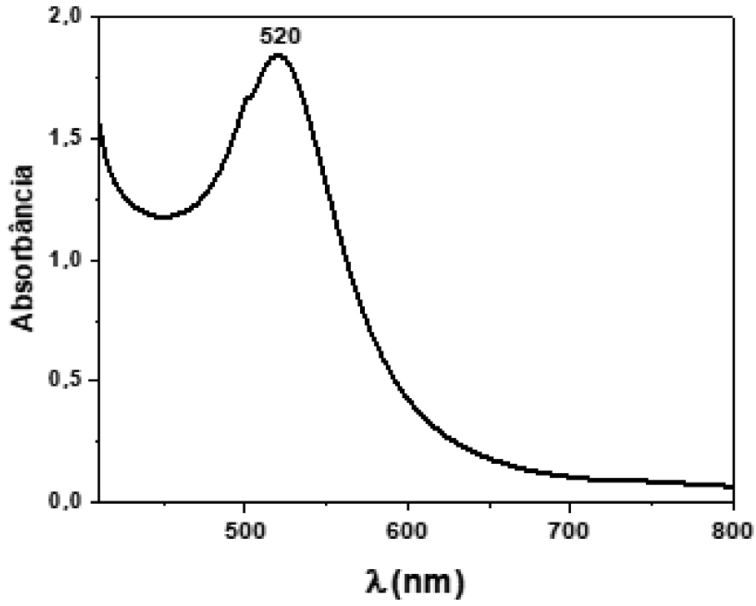

Figura 2. Espectros de absorção do coloide de AuNPs sintetizado neste trabalho

origem no espalhamento de luz provocado pelas nanopartículas em suspensão, sendo um teste simples e que também pode ser realizado no decorrer da síntese das nanopartículas. ${ }^{22}$ Assim, a visualização do caminho óptico do laser é um indicador da presença de nanopartículas suspensas no solvente.
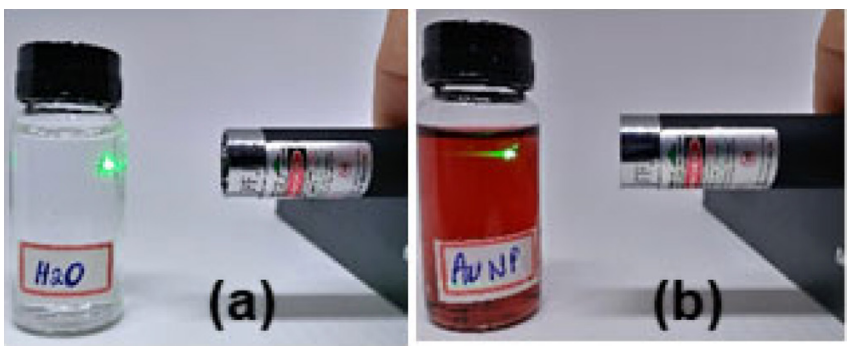

Figura 3. Demonstração do efeito Tyndall com a incidência de um feixe de luz laser através de um recipiente contendo água destilada (a) e da amostra de AuNPs (b)

Na Figura 4(a)-(c) são mostradas imagens de TEM do coloide de AuNPs, em diferentes magnificações, em que é possível observar nanopartículas com morfologia esferoidal. Com o aumento da magnificação é possível observar mais detalhes das partículas, que apresentam homogeneidade na aparência física. Esse resultado mostra que o método de síntese empregado permitiu obter uma amostra homogênea de AuNPs. Realizando a contagem do diâmetro das nanopartículas de ouro em diferentes imagens de TEM, obteve-se uma distribuição de tamanho que apresentou bom ajuste a função gaussiana, com valor médio de 13,6 nm, Figura 4(d).

Embora a discussão do mecanismo de formação das AuNPs pela redução por citrato de sódio esteja fora do escopo do artigo, apresentamos as duas principais etapas baseadas em evidências experimentais e teóricas já publicadas. ${ }^{23,24}$ Inicialmente, o $\mathrm{Au}^{3+}$ é reduzido a $\mathrm{Au}^{+}$, como mostrando na equação química (1). $\mathrm{Na}$ segunda etapa, equação química (2), a espécie química $\left[\mathrm{Au}^{+}\left(\mathrm{Cl}^{-}\right)_{2}\right]^{-}$ sofre desproporcionamento, formando átomos de ouro e o complexo tetracloreto de ouro(III). Finalmente, os átomos de ouro formados se ligam entre si, dando origem as nanopartículas que são estabilizadas pelos ânions citratos. De acordo com Ojea-Jimenez, ${ }^{24}$ outras etapas são possíveis no mecanismo de formação das AuNPs em função do $\mathrm{pH}$ do meio. As etapas apresentadas nas equações químicas (1) e (2), estão de acordo com o mecanismo em meio ácido, já que no presente trabalho o $\mathrm{pH}$ do meio reacional variou de 4,0, no início da reação, para 6,3, no coloide final. Essa variação de pH está de 

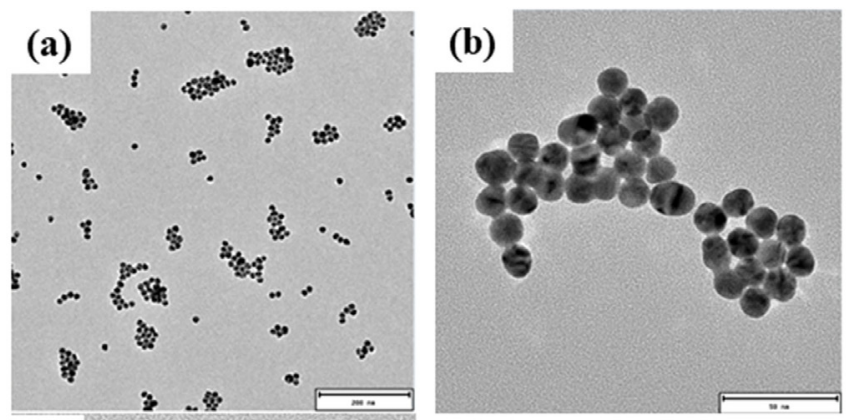

(c)
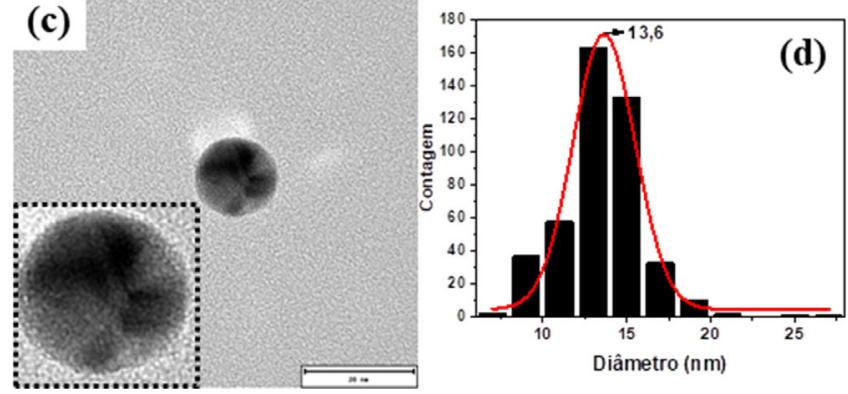

Figura 4. Imagens representativas de TEM do coloide de nanopartículas de ouro em diferentes magnificações e escalas de tamanho $200 \mathrm{~nm}$ (a), 50 $\mathrm{nm}$ (b) e $20 \mathrm{~nm}$ (c). Histograma de distribuição de tamanho das AuNPs (d)

acordo com a redução da concentração do ácido cloroáurico, que é consumido na reação.

$\left[\mathrm{Au}^{3+}\left(\mathrm{Cl}^{-}\right)_{4}\right]^{-}+\left[\mathrm{C}_{6} \mathrm{H}_{7} \mathrm{O}_{7}\right]^{-} \rightarrow \mathrm{CO}_{2}+2 \mathrm{Cl}^{-}+\left[\mathrm{Au}^{+}\left(\mathrm{Cl}^{-}\right)_{2}\right]^{-}+\mathrm{C}_{5} \mathrm{H}_{6} \mathrm{O}_{5}+$ $\mathrm{H}^{+}$

$3\left[\mathrm{Au}^{+}\left(\mathrm{Cl}^{-}\right)_{2}\right]^{-} \rightarrow 2 \mathrm{Au}^{\mathrm{o}}+\left[\mathrm{Au}^{3+}\left(\mathrm{Cl}^{-}\right)_{4}\right]^{-}+2 \mathrm{Cl}^{-}$

(Ilustração das principais etapas do mecanismo de formação de nanopartículas de ouro na presença do ânion citrato)

Antes de iniciar os testes colorimétricos do sulfatiazol usando as AuNPs preparadas, a solução mais concentrada do composto foi analisada por espectrofotometria e o espectro de absorção registrado é apresentado na Figura 5. É possível observar na Figura 5, as duas bandas intensas características do sulfatiazol, com absorção na região do ultravioleta em 260 e 285 nm, de acordo com trabalho já publicado. ${ }^{25} \mathrm{O}$ conhecimento do espectro de absorção do sulfatiazol foi importante para o teste de detecção discutido na sequência, já que o composto apresenta absorções em região distinta do coloide de AuNPs.

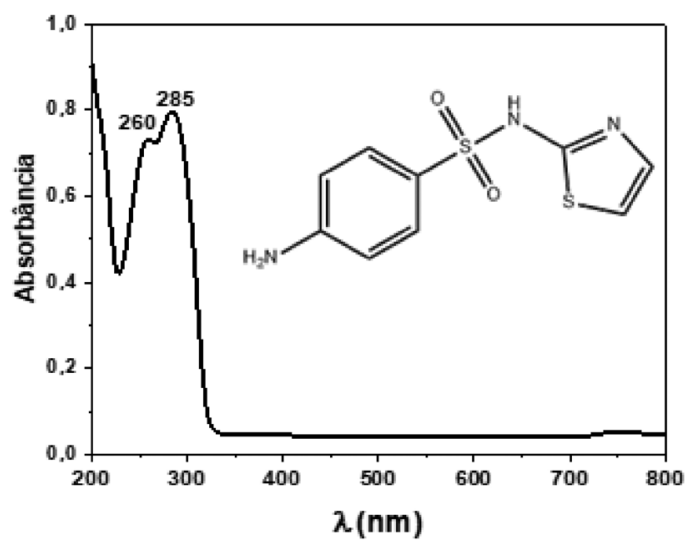

Figura 5. Espectro de absorção do sulfatiazol na concentração $2,0 \times 10^{-3} \mathrm{~mol} \mathrm{~L}^{-1}$ e sua estrutura molecular
Na Figura 6 são apresentados os resultados do teste de detecção colorimétrica do sulfatiazol usando as AuNPs, que também foi monitorado por espectrofotometria de absorção molecular.
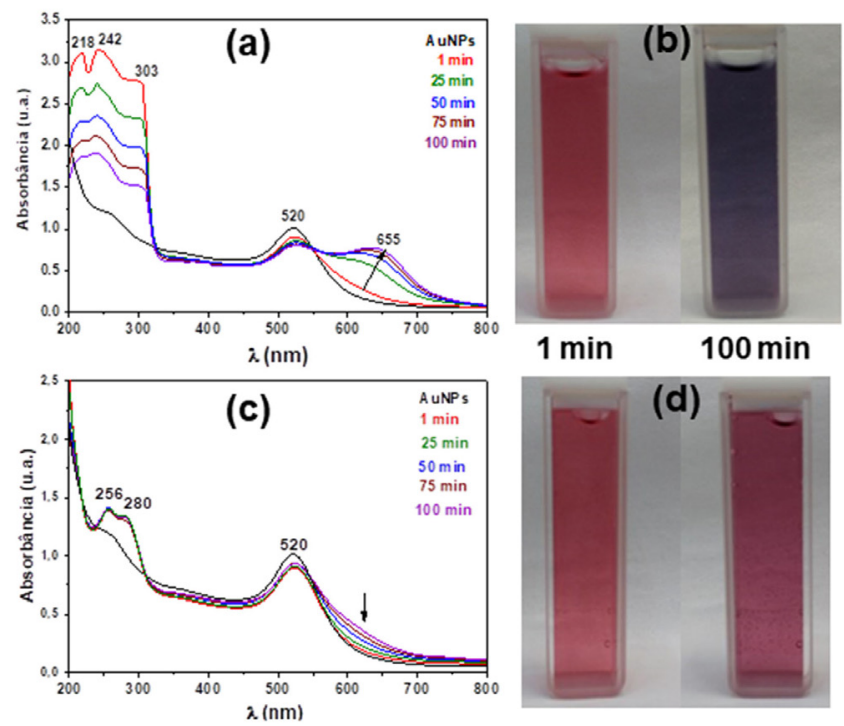

Figura 6. Espectros de absorção do coloide de AuNPs antes e após a adição da solução de sulfatiazol 2,0 $\times 10^{-3} \mathrm{~mol} \mathrm{~L}^{-1}$ para diferentes tempos (a) e fotografias da cubeta de quartzo contendo a mistura do coloide de AuNPs e a solução de sulfatiazol nos tempos de 1 e 100 min (b). Espectro de absorção do coloide de AuNPs antes e após a adição da solução de sulfatiazol 1,0 $\times 10^{-4} \mathrm{~mol} \mathrm{~L}^{-1}$ para diferentes tempos (c) e fotografias da cubeta de quartzo contendo a mistura do coloide de AuNPs e a solução de sulfatiazol nos tempos de 1 e 100 min (d)

Na Figura 6(a), é mostrado o espectro de absorção do coloide de AuNPs puro e após a adição da solução de sulfatiazol 2,0 x $10^{-3} \mathrm{~mol} \mathrm{~L}^{-1}$, concentração final $5,7 \times 10^{-4} \mathrm{~mol} \mathrm{~L}^{-1}$, no primeiro minuto até 100 min. A cubeta só foi removida do espectrofotômetro após este tempo, sendo observada a mudança de cor do vermelho para o violeta, servindo como indicador colorimétrico da interação das moléculas de sulfatiazol com as AuNPs, Figura 6(b). Ao analisar detalhadamente o conjunto de espectros de absorção do teste, observa-se o surgimento de uma segunda banda SPR em $655 \mathrm{~nm}$, em função do tempo, sendo indicativa da formação de agregados das AuNPs induzido pela presença do sulfatiazol. Essa interpretação também corrobora com as discussões na literatura científica sobre a formação de agregados de nanopartículas de ouro. ${ }^{26,27} \mathrm{O}$ espalhamento de luz é diferente quando as nanopartículas estão separadas em comparação com agregados, resultando em mudanças na coloração observada. ${ }^{27}$ Tomando vantagem desse efeito, à mudança de cor do meio pode ser usada como um indicador colorimétrico da presença das moléculas de sulfatiazol.

O teste de detecção para a solução mais diluída do sulfatiazol, concentração final $2,9 \times 10^{-5} \mathrm{~mol} \mathrm{~L}^{-1}$, apresentou pouca variação dos espectros de absorção após 25 min e uma suave mudança na cor do coloide, Figura 6(c) e (d). Apesar dos efeitos serem menos pronunciados, esse resultado indica que ainda é possível monitorar a presença de sulfatiazol no meio aplicando a mesma metodologia. Adicionalmente, esse último resultado também sugere que nesta condição o teste atingiu o seu limite de detecção. Vale ressaltar ainda, que em ambos os testes as bandas do sulfatiazol também são observadas na região UV dos espectros, Figura 6(a) e (c). Porém, devido à aleatoriedade das posições, apenas a banda SPR das AuNPs foi usada como indicador do sensoriamento devido a consistência do sinal.

Com base no resultado do teste colorimétrico discutido acima, é proposto o desenho da Figura 7, como uma ilustração didática 
para o melhor entendimento do fenômeno em nível molecular. Na condição inicial, as nanopartículas de ouro estão dispersas no solvente e se mantém afastadas por repulsão eletrostática, devido à presença de cargas negativas dos ânions citrato que estão ligados na superfície das AuNPs atuando como agente estabilizante. A formação de agregados é induzida pela presença de moléculas de sulfatiazol, levando à mudança de cor do meio. A molécula de sulfatiazol apresenta grupos funcionais com átomos de enxofre e nitrogênio, que possuem pares de elétrons livres, e podem se coordenar aos átomos de ouro na superfície das nanopartículas. ${ }^{28}$ Como as interações Au-S e Au-N são mais efetivas do que Aucitrato, sugere-se que a interação ocorre com liberação de ânions citrato para a solução, ocorrendo a troca de estabilizantes antes na superfície das AuNPs. Além disso, as moléculas de sulfatiazol podem se ligar à superfície de mais de uma nanopartícula, atuando como uma ponte e aproximando as AuNPs provocando a formação do agregado. De acordo com o observado na Figura 6 e discutido acima, a formação de agregados é dependente da concentração da solução de sulfatiazol. Essa interpretação e modelo de formação de agregados das AuNPs na presença de sulfatiazol está de acordo com relatos de trabalhos que estudaram em detalhes esse fenômeno para nanopartículas de ouro em contato com várias moléculas e sais que induziram agregação. ${ }^{26,27}$

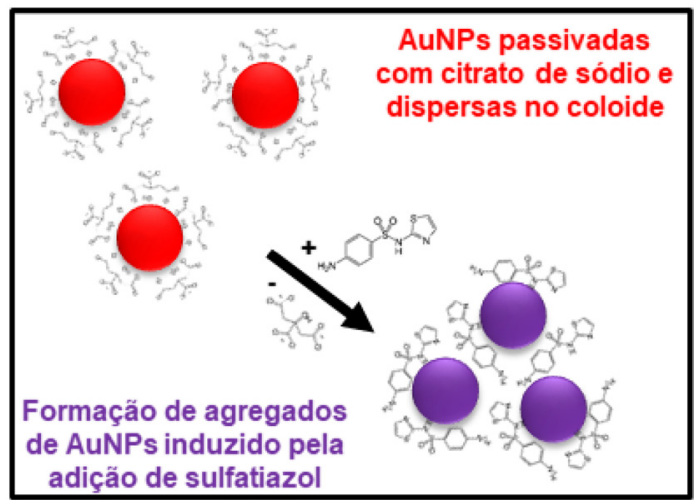

Figura 7. Esquema ilustrativo da interação das moléculas de sulfatiazol com as nanopartículas de ouro resultando em agregação e mudança de cor do coloide de AuNPs

Na Tabela 1 são descritas algumas questões de aprendizagem que podem ser abordadas pelo docente ao longo das três aulas experimentais. As questões foram selecionadas de acordo com os conteúdos teóricos mais importantes de cada etapa. Por exemplo, a síntese das AuNPs ocorre via reação de redução dos íons $\mathrm{Au}^{3+}$

Tabela 1. Questões de aprendizagem para cada etapa da prática experimental

\begin{tabular}{cc}
\hline Procedimento & Questões de aprendizagem \\
\hline Síntese das AuNPs & - Preparo de soluções químicas. \\
& - Reação de óxido-redução. \\
& - Balanceamento de reações. \\
- Química coloidal.
\end{tabular}

para átomos de $\mathrm{Au}^{0}$, os quais agregam gerando as AuNPs. Com esse procedimento, o docente poderá trabalhar com os estudantes vários tópicos de química, como descrito na Tabela 1. Nas duas etapas seguintes, alguns tópicos são mais avançados e/ou interdisciplinares, como por exemplo, as técnicas de caracterização, classe de fármacos, contaminantes emergentes, espalhamento de luz, etc. Essas questões podem ser escolhidas de acordo com a ênfase que o docente deseja direcionar a prática, o nível de aprofundamento dos assuntos, o curso de formação dos estudantes e o perfil da turma, graduação ou pós-graduação. Além disso, o docente também poderá propor outras questões de aprendizagem que considerar relevantes.

\section{CONCLUSÕES}

No presente trabalho, foi realizada a síntese de AuNPS usando citrato de sódio como agente redutor e estabilizante, com tamanho médio igual a 13,6 nm e banda SPR em $520 \mathrm{~nm}$. As AuNPs exibiram cor vermelha quando estavam dispersas no solvente, mudando para cor violeta após adição de solução de sulfatiazol. Essa mudança de coloração ocorreu devido à formação de agregados de AuNPs induzidos pelas moléculas de sulfatiazol. $\mathrm{O}$ fenômeno foi facilmente monitorado por espectrofotometria de absorção e inspeção visual do recipiente, podendo ser adotado como teste colorimétrico para detecção de sulfatiazol. Devido à facilidade de execução e caráter interdisciplinar, o presente trabalho foi apresentado como uma proposta de prática experimental para aulas com foco em nanotecnologia e interface com outras disciplinas, como por exemplo, química, física e ciência de materiais.

\section{MATERIAL SUPLEMENTAR}

Outras imagens de microscopia eletrônica de transmissão do coloide de nanopartículas de ouro preparado neste trabalho estão livremente acessíveis em http://quimicanova.sbq.org.br/.

\section{AGRADECIMENTOS}

Os autores agradecem à FAPESP (Processo 2013/22127-2, 2014/50906-9 e 2018/07136-9) e ao CNPq (310131/2020-0 e 405087/2021-7) pelo apoio financeiro. Os autores também agradecem ao Laboratório Nacional de Nanotecnologia (LNNano-CNPEM), pelo acesso ao microscópio eletrônico de transmissão.

\section{REFERÊNCIAS}

1. Santos, V. M. C.; Frigeri, H. R.; Resumos do XI Congresso Nacional de Educação, Curitiba, Brasil, 2013.

2. Lima, J. O. G.; Alves, I. M. R.; Rev. Bras. Ens. Ci. Tecnol. 2016, 9, 428.

3. Silva, A. X.; Cusati, I. C.; Guerra, M. G. G. V.; Rev. Ibero-Am. Estud. Edu. 2018, 13, 979.

4. https://www.un.org/sustainabledevelopment, acessada em janeiro 2022.

5. Porter, A. L.; Youtie, J.; J. Nanopart. Res. 2009, 11, 1023.

6. Mulvaney, P.; ACS Nano 2015, 9, 2215.

7. Jeevanandam, J.; Barhoum, A.; Chan, Y. S.; Dufresne, A.; Danquah, M. K.; Beilstein J. Nanotechnol. 2018, 9, 1050.

8. Silva, A. G. M.; Rodrigues, T. S.; Macedo, A.; Silva, R. T. P.; Camargo, P. H. C.; Quím. Nova 2014, 37, 1716.

9. Crut, A.; Maioli, P.; Fatti, N. D.; Vallee, F.; Chem. Soc. Rev. 2014, 43, 3921.

10. Ogarev, V. A.; Rudoi, V. M.; Dementeva, O. V.; Inorg. Mater. Appl. Res. 2018, 9, 134.

11. Finch, R.; Greenwood, D.; Whitley, R.; Norrby, S. R.; Antibiotic and Chemotherapy, 9th ed., Elsevier Saunders: Edinburgh, 2010. 
12. Leston, S.; Nebot, C.; Nunes, M.; Cepeda, A.; Pardal, M. A.; Ramos, F.; Environ. Toxicol. Pharmacol. 2015, 39, 77.

13. Lin, X.; Xu, J.; Keller, A. A.; He, L.; Gu, Y.; Zheng, W.; Sun, D.; Lu, Z.; Huang, J.; Huang, X.; Li, G.; Sci. Total Environ. 2020, 744, 140977.

14. Ratkaj, M.; Miljanic, S.; Vib. Spectrosc. 2014, 74, 104.

15. Sun, Y.; Gao, H.; Xu, L.; Waterhouse, G. I. N.; Zhang, H.; Qiao, X.; Xu, Z.; Food Chem. 2020, 332, 127376.

16. Ozin, G. A.; Arsenault, A. C.; Ludovico, Cademartiri, L.; Nanochemistry: A chemical approach to nanomaterials, $2^{\text {nd }}$ ed., RSC Publishing: Cambridge, 2009.

17. Zhao, L.; Jiang, D.; Cai, Y.; Ji, X.; Xie, R.; Yang, W.; Nanoscale 2012, 4, 5071.

18. Liaw, J.; Tsai, S.; Lin, H.; Yen, T.; Chen, B.; J. Quant. Spectrosc. Radiat. Transf. 2012, 113, 2234.

19. Anjum, D. H.; IOP. Conf. Ser.: Mater. Sci. Eng. 2016, 146, 012001.

20. Ferrari, T. C.; Menechini Neto, R.; Santos, O. A. A.; Jorge, L. M. M.; Braz. Appl. Sci. Rev. 2019, 3, 2718.
21. Rojas, C. E. B.; Martins, A. H.; R. Esc. Minas 2010, 63, 315.

22. Deng, Z.; Jin, W.; Yin, O.; Huang, J.; Huang, Z.; Fu, H.; Yuan, Y.; Zou, J.; Nie, J.; Zhang, Y.; Chem. Commun. 2021, 57, 2613.

23. Ojea-Gimenez, I.; Romero, F. M.; Bastus, N. G.; Puntes, V.; J. Phys. Chem. C 2010, 114, 1800.

24. Ojea-Gimenez, I.; Campanera, J. M.; J. Phys. Chem. C 2012, 116, 23682.

25. Zhu, G.; Sun, Q.; Wang, C.; Yang, Z.; Xue, Q.; Int. J. Environ. Res. Public Health 2019, 16, 1797.

26. Zakaria, H. M.; Shah, A.; Konieczny, M.; Hoffmann, J. A.; Nijdam, A. J.; Reeves, M. E.; Langmuir 2013, 29, 7661.

27. Xu, X.; Ji, J.; Chen, P.; Wu, J.; Jin, Y.; Zhang, L.; Du, S.; Anal. Chim. Acta 2020, 1125, 41.

28. Reimers, J. R.; Ford, M. J.; Halder, A.; Ulstrup, J.; Hush, N. S.; Proc. Natl. Acad. Sci. U. S. A. 2016, 113, 1424. 